\title{
PRAGMÁTICA FRENTE A GESTIÓN DE INFORMACIÓN: CRITICA DE THE SEMANTIC STRUCTURE OF SPANISH DE LARRY KING
}

Joaquín Garrido Medina. Universidad Complutense de Madrid.

\section{INTRODUCCIÓN: RASGOS SEMÁNTICOS Y MORFOLOGíA FLEXIVA.}

El libro de Larry King The semantic structure of Spanish: meaning and grammatical form ' constituye un acontecimiento agradable: no se puede menos que dar la bienvenida a un estudio semántico del español escrito en inglés en Estados Unidos (King pertenece a la Universidad de Carolina del Norte) $y$ publicado en una editorial holandesa (y estadounidense). Se trata de un estudio de morfología flexiva verbal, principalmente, desde el punto de vista del significado: tiempo, aspecto y modo verbales ocupan cinco capitulos (segundo a sexto), mientras que se dedican sendos capítulos (séptimo y octavo) a la determinación y el número nominal, por una parte, y el uso de preposición $a$ con complemento directo, por la otra. Al estudio del español se añade un capítulo (el noveno) de comparación con el inglés, y un último capítulo (décimo) de conclusiones. El título del libro hace referencia al hecho de que el análisis se lleva a cabo mediante rasgos semánticos binarios ("estructura semántica"), aplicado a la morfología en sentido amplio ("significado y forma gramatical"). Son antecedentes directos del libro algunos artículos del autor ${ }^{2}$ o del autor y Margarita Suñer ${ }^{3}$.

1 Amsterdam y Nueva York, Benjamins, 1992.

2 "The semantics of tense, orientation and aspect in English", Lingwa, 59, 1983, págs. 101-154; "The semantics of direct object $a$ in Spanish", Hispania, 67, 1984, páginas $397-404$.

s "The meaning of the progressive in Spanish and Portuguese", The Bilingual Reviezv, 7, 1980, págs. 228-238; "On the notion of stativity in Spanish and Portuguese", en 
Hay un problema que se suscita desde la primera página: King se sitúa al margen de muchos estudios llevados a cabo sobre su tema principal, el verbo español (mencionemos como ejemplo la antolugia de Gierd Wotjak y Alexandre Veiga ${ }^{4}$ y las dos de Ignacio Bosque ${ }^{5}$, dos años anteriores a la obra de King). Por ejemplo, para defender la idea de que no se ha prestado atención al "significado de la forma gramatical", afirma en el prefacio que el significado oracional y el del enunciado se han analizado en obras de Chomsky, Partee y Grice, todas de los años sesenta. Las antologías de P. J. Tedeschi y Annie Zaenen ${ }^{6}$ y de P. J. Hopper ${ }^{7}$, además de contradecir la afirmación de King, son muestras de ausencias sintomáticas. Aparece, por ejemplo, añadida marginalmente en nota $9.3,252)$, la mención al análisis de Hans Reichenbach $^{8}$ aplicado al español por Karen Zagona ${ }^{9}$, pero no aparece el propio Reichenbach ni otras versiones como la de Juan José Acero ${ }^{10} \mathrm{o}$ la de quien esto escribe ${ }^{11}$. Tampoco es justa la afirmación de que en las obras de gramática generativa no se ha tenido en cuenta la flexión verbal, como prueba el estudio de Elena de Miguel $^{12}$ sobre el aspecto en español, posterior a la redacción de la obra de King: o, en la gramática de Montague, la obra de David Dowty ${ }^{13}$, que incorpora ideas de Reichenbach, y su aplicación al español por parte de Juan Carlos Moreno Cabrera ${ }^{14}$. Notemos como muestra de que

Frank H. Nuessel (ed.), Contemporary Studies in Romance Languages, Bloomington, Indiana University Linguistics Club, 1980, págs. 183-201. En previsión de una posible traducción al español de la obra, las citas del libro de King remitirán al capítulo y apartado, por ejemplo 3.3, seguidos de la página: $3.3,20$.

- La descripción del verbo español. Santiago de Compostela, Universidad, 1990, Anejo 32 de Verba.

5 Indicativo y subjuntivo, Madrid, Taurus, 1990; Ticmpo y aspecto en español, Madrid. Cátedra, 1990.

- Syntax and scmantics, vol. 14: Tense and aspect, Nueva York, Academic Press, 1981.

7 Tense and aspect: lictween semantics and pragmatics, Amsterdam. Benjamins, 1982.

8 Elements of symbolic logic, Londres, Macmillan, 1947, § 51.

9 Verb phrase syntax, A parametric study of Fnglish and Spanish. Dordrecht, Kluwer (citada como Zargona), 1988.

${ }_{10}$ "Las ideas de Reichenbach acerca del tiempo verbal", en I. Bosque (ed.), Tiempo $y$ aspecto en español, Madrid, Cátedra, 1990, págs. 45-75.

i1 J. Garrido, Lógica y lingiuistica, Madrid, Síntesis, 1988, \& 4.3.2.

12 El aspecto en la sintaxis del español. Madrid, Universidad Autónoma de Madrid, 1992.

13 Studics in the logic of verb aspect and time refercnce in English, Austin, Universidad de Tejas, 1972; Word meaning and Montague grammar. Dordrecht, Reidel, 1979, y "Tenses, time adverbs and compositional semantic theory". Linguistics and Philosophy, 5, 1982, págs. 23-55.

14 "Lógica temporal, semántica de intervalos y análisis lingüistico del tiempo y aspectos verbales", en las Actas del II Congreso de Lenguajes Naturales y Lenguajes For. males, Barcelona, PPU, 1987, págs. 93-112. 
Reichenbach sigue vigente el artículo de Ángeles Carrasco ${ }^{15}$.

Al tratar las teorias del significado existentes, en el primer capítulo, King encuentra dos. La primera es la que llama jakobsoniana, que ejemplifica con el análisis del verbo francés realizado por Linda Waugh ${ }^{16}$. Es de justicia contradecir la afirmación de King acerca de la falta de estudios sobre el significado en la morfología flexiva: baste mencionar el clásico estudio de Roman Jakobson ${ }^{17}$ sobre el verbo ruso (King solo cita genéricamente sus obras escogidas de 1971) o el estudio de los casos de Louis Hjelmslev ${ }^{18}$, quien precisamente aplica con claridad la diferencia entre una categoría A y otra categoría constituida por A y no A.

La segunda teoría semántica mencionada por King es la de prototipos de Eleonor Rosch y cognoscitiva de George Lakoff y Ronald Langacker ${ }^{19}$. Sin embargo, a pesar de su adscripción a esta teoría, cuando King entra en materia, emplea la teoria "jakobsoniana", tras revisar los análisis sobre el tiempo verbal de Gili Gaya, Bull y Stockwell, Bowen y Martin ${ }^{20}$. E inmediatamente puede surgir la sorpresa en el ánimo del lector: la sorpresa de encontrar y no encontrar la huella de Hjelmslev y Alarcos.

Antes de seguir conviene observar la meticulosa composición del libro, ordenado en capítulos y apartados con títulos que facilitan la lectura, provisto de útiles indices de autores y términos, y con una bibliografia sin errores ni omisiones.

15. "Problemas para un análisis neoreichenbachiano de algunos hechos de concordancia temporal", ('uadernos de Lingüística, 1, 1993.

16 "A semantic analysis of the French tense system", Orbis, 25, 1975, págs. 436-485.

17 "Zur Struktur des russischen Verbums", en R. Jakobson, Selected writings, 2, La Haya, Mouton, págs. 3-15.

18 La categoria de los casos (1935), Madrid, Gredos, 1978, § I.A.2, pág. 142.

19 Eleanor Rosch, "Cognitive representations of semantic categories", Journal of Experimental Psychology: General, 104, 1975, págs. 192-233; George Lakoff, Women, fire, and dangerous things, Chicago y Londres, University of Chicago Press, 1987; Ronald W. Langacker, Foundations of cognitive grammar, primera edición, Tréveris, Linguistic Agency University of Trier, 1983; segunda edición, Stanford, Stanford University Press, 1987.

20 Samuel Gili Gaya, Curso superior de sintaxis española, primera edición, 1943; segunda edición, Barcelona, 1961; reimpresión, 1973, Biblograf; William E. Bull, Time, tense, and the verb: $A$ study in theoretical and applied linguistics, with particular attention to Spanish, Berkeley y Los Angeles, University of California Press, 1960; Robert P. Stockwell, J. Donald Bowen y John W. Martin, The grammatical structures of English and Spanish, Chicago, University of Chicago Press, 1965. 


\section{EL TIEMPO.}

\subsection{Perspectivas en el presente, el imperfecto y el futuro.}

En el capítulo tercero (3.3, 20), King propone su tesis: el tiempo verbal ("tense") consiste en la "organización del tiempo basada en perspectivas, no en puntos del tiempo". King llama "tiempo de la comunicación" al tiempo de la enunciación (aqui empleo yo el término de Reichenbach ${ }^{21}$ ), y se refiere a él con las siglas inglesas TOC (según una costumbre terminológica muy extendida). Más que definirla, introduce la perspectiva como sigue $(3.2,19)$ :

El tiempo de comunicación $[\ldots]$ hace referencia a la perspectiva subjetiva desde la cual observa el hablante toda la realidad (pasada, presente y futura).

Presente, imperfecto y futuro de indicativo quedan asi caracterizados con los correspondientes rasgos semánticos:

$$
\begin{aligned}
& \text { [+ presente] PERSPECTIVA TEMPORAL PRESENTE }(3.3,23) \\
& \text { [+ pasado] PERSPECTIVA TEMPORAL PASADA }(3.4,24) \\
& \text { [+ futuro] PERSPECTIVA temporal futura }(3.4,27) \text {. }
\end{aligned}
$$

Como ejemplo de su definición, veamos la del rasgo semántico de futuro: "significa que la situación descrita por el verbo se extrae de la perspectiva presente y se asocia a la perspectiva futura" $(3.4,27)$.

Hay además otra perspectiva, que explica los usos que no encajan en este análisis; para el presente ( $L a$ independencia se declara en 1776, Me marcho mañana) es "la perspectiva de hecho" $(3.4,23)$, y para el futuro (Eistará en casa) es la perspectiva "predictiva" $(3.4,27)$. En estos casos, según King, "el sistema verbal explota pragmáticamente hasta agotarla la oposición entre presente como hecho y futuro como predicción" $(3.4,27)$. Notemos de pasada que King se refiere al análisis de Dwight Bolinger ${ }^{22}$ del presente en inglés, pero bien se hubiera podido basar en la observación de Andrés Bello ${ }^{23}$, de que "son [las cuatro] denotará certidumbre, y serán [las cuatro] cálculo, raciocinio, conjetura", o en la de Amado Alonso y Pedro Henríquez Ureña ${ }^{24}$, según la cual "la forma del presente añade una resolución o convicción o seguridad actual de que el hecho ocurrirá”.

\footnotetext{
2 Véase la nota 8.

2 "More on the present tense in English", Langwage, 23, 1947, págs. 434-436.

23 Gramática de la lengwa castellana destinada al uso de los americanos, primera edición, 1847; cuarta y última edición del autor, 1860; Madrid, Arco, 1989, \& 689.

* Gromática castellana, segundo curso, primera edición, 1939, Buenos Aires, Losada, 1977, \& 192.
} 
Análogamente a la oposición entre presente y futuro, en $Y_{O}$ era la princesa, según King "se permite al hablante evitar la descripción muy objetiva de la realidad que sería implicada con la forma del presente (Yo soy la princesa)", es decir, "se permite al hablante manipular pragmáticamente la perspectiva temporal y la referencia temporal del mundo real" $(3.4,26)$. En Quería pedirle un favor, con el imperfecto se evita el "tono fáctico" del presente quiero: "la interpretación pragmática de cortesía proviene del significado de la forma pasada en oposición al significado de la forma presente" $(3.4,25)$.

\subsection{Semántica frente a pragmática; el condicional.}

Aparece así una concepción de lo pragmático frente a lo semántico que consiste simplemente en el uso frente al sistema, el habla frente a la lengua; efectivamente, el análisıs de Gili Gaya es para King "un inventario de posibilidades pragmáticas" $(3.1,17)$, mientras que su propio análisis "tiene en cuenta los usos pragmáticos apropiados de las formas gramaticales" $(3.6,35)$. Como el estudio es de carácter semántico, en el apartado dedicado a la semántica y pragmática del tiempo verbal, "las implicaciones/inferencias potenciales de los significados invariantes simplemente se enumeran" $(3.6,38)$. Aunque King mantiene que su análisis "explica la motivación semántica existente tras un determinado uso pragmático" $(3.7,41)$, no queda claro en qué se diferencia su análisis, por ejemplo, del de Bello ${ }^{25}$ acerca del presente histórico como sustitución metafórica de la relación de anterioridad por la de coexistencia para expresar "con más viveza los recuerdos". El propio Bello ${ }^{20}$ distingue en cada tiempo verbal "además de su valor propio y fundamental, otros diferentes en que se convierte el primero según ciertas reglas generales".

$\mathrm{El}$ condicional es la "forma atemporal"; su "significado invariante" es "PERSPECtiva ATEMPORAL (es decir, sin perspectiva en absoluto, la perspectiva intemporal), y se le asigna el rasgo [- temporal]" (pág. 29). De nuevo puede venir a la memoria del lector el curso de Alonso y Henríquez Ureña ${ }^{27}$, según el cual en tendría "no hay propiamente expresión de tiempo", sino de "una posibilidad que cabe en cualquier época". Dejemos de lado la afirmación de King $(3.5,28)$ de que Bello considera el condicional como modo diferente, que denomina "subjuntivo hipotético", puesto que basta releer los párrafos en que Bello insiste en que el "pos-pretérito" cantaría pertenece al

20 Gramática, § 686.

* Gramática, \& 620.

27 Gramática española, segundo curso, \& 197. 
indicativo, y en que denomina "subjuntivo hipotético" a canlare y hubicre cantado 28 .

King se basa en los ejemplos de Gili Gaya ${ }^{2}$, Dijo que vendria: $y$ vino, $y$ viene en efecto, $y$ le esperamos, para negar referencia temporal al conclicional. Sin embargo, no tiene en cuenta la evidente observación de Gili Gaya ${ }^{30}$ acerca de que "la relación con el presente [de la enunciación] es variable, en tanto que su relación con el pretérito es fija"; "es futuro desde el pasado". Para King, Estariamos aqui y Serian las diez expresan predicciones acerca del presente y del pasado, respectivamente, "ya que cualquier situación presentada como hipotética y sin asociar a perspectiva temporal alguna deberia suponer especulación, que se podría interpretar contextualmente como predicción" $(3.5,30)$. Igualmente, Seria un buen padre se explica porque "el significado invariante de [- temporal] siempre describe la situación como hipotética en el momento de habla" $(3.5,32)$.

$\mathrm{Si}$ "siempre" que se usa una expresión aparece una cierta información, ¿no forma parte de su significado? Las perspectivas de fáctico, predictivo e hipotético son en la práctica otros rasgos. Para explicar Si tuviera dinero compraba esta casa, que Gili Gaya ${ }^{31}$ atribuye al carácter de acción inacabada del imperfecto, de manera que se sobrepone el aspecto al tiempo, King aduce "la perspectiva no presente y por lo tanto no fáctica del pasado" $(3.5,33)$. Vemos pues que para explicar ciertos usos de los tiempos verbales, King opone los demás tiempos al carácter fáctico característico del presente: de hecho, King está proponiendo que el presente es el término marcado (frente a los otros tiempos que no están marcados como fácticos). La parte de su análisis que considera pragmática es en realidad tan semántica como la otra; se puede resumir como sigue:

fáctico: presente

no fáctico: imperfecto, futuro, condicional.

En la opción entre Me dijo que fuiste al cine anoche y Me dice que fuiste al cine anoche, King afirma que el uso del presente "no es predecible"; "el contexto es crucial" $(3.7,40)$. Sin embargo, su concepción del contexto es extralingüística : el contexto es "una circunstancia concreta (del mundo real) en la cual uno se encuentra en un determinado momento", de manera que los contextos no se pueden enumerar $(3.7,40)$. El razonamiento de King parece circular: lo que determina la elección del tiempo verbal es la perspectiva del hablante, pero precisamente eso, la perspectiva del hablante, es lo que según

\footnotetext{
28 Gramática, $\S 453$ y nota 14, y $\S 658$, respectivamente.

29 Curso, \& 129.

30 Curso, \& 129.

s1 Curso, \& 124.
} 
King expresa el tiempo verbal. Con la única limitación de que el tiempo elegido sea compatible con el contexto (es decir, para King, con la situación extralingüistica), el hablante puede elegir cualquier perspectiva, afirma King.

En realidad, King propone dos tipos de rasgos. A la diferencia entre fáctico $y$ no fáctico que King considera pragmática, se añade la diferencia entre temporalidad y no temporalidad, la única que para King es semántica:

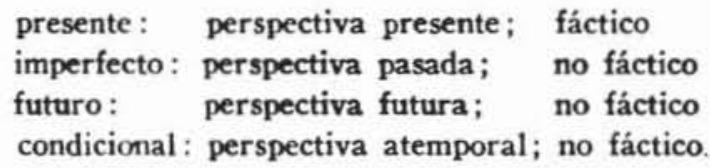

Tengamos en cuenta que King $(5.6,37)$ jerarquiza los rasgos de manera que el presente está marcado mientras que el imperfecto y el futuro no están marcados en cuanto a la perspectiva de presente, y el imperfecto está marcado con la perspectiva pasada frente al futuro (aunque el rasgo de no marcado como pasado se denomina [ + futuro], es decir, marcado para el futuro); los tres están marcados por la temporalidad frente al condicional. Añadiendo (a la izquierda) el rasgo del carácter fáctico, que marca al presente frente a los demás tiempos, obtenemos el siguiente esquema:

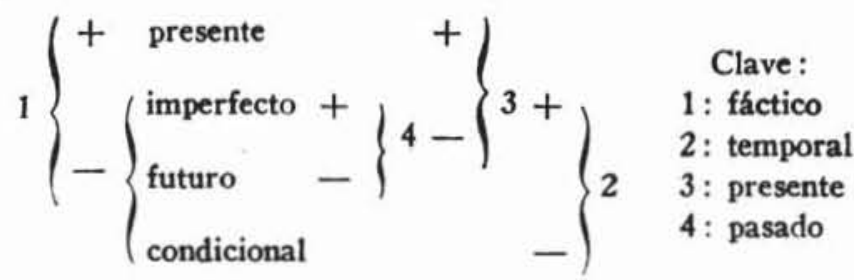

Visto asi, con el rasgo de fáctico que marca al presente, el análisis de King no parece tan sistemático como haría pensar el término de "semántica sistemática" que propone (en $3.6,37$ ) para destacar la semejanza que encuentra entre la estructura semántica y la fonológica. (Ante esta analogía, el lector, ya que King no lo hace, recordará el antecedente de la propuesta metodológica de Hielmsley ${ }^{32}$, desarrollada en la obra de Coseriu ${ }^{33}$.)

\subsection{Reminiscencias del pasado: Hjelmslev y Alarcos de incógnito.}

King mantiene que su uso de perspectivas es una idea de la lingüística cognoscitiva $(3.6,34)$, aunque en mi opinión el enfoque no es cognoscitivista

\footnotetext{
3 Louis Hjelmslev, "Para una semántica estructural" (1957), en L. Hjelmslev, Ensayos lingüísticos (1959), Madrid, Gredos, 1972, págs. 125-146.

ss Eugenio Coseriu, "Introducción al estudio estructural del léxico" (1964), en E. Coseriu, Principios de semántica estructural, Madrid, Gredos, 1977, págs. 87-142.
} 
en la medida en que las nociones que emplea el hablante propuestas por King son según él "abstractas" en el sentido de "sin relacionar con el mundo real tal como es percibido" $(3.6,35)$; en nota indica King $(3.6,34, \mathrm{n} .6)$ que la perspectiva del hablante no es nueva en lingüística.

Efectivamente, en la gramática que Alarcos subtitula explícitamente "según la escuela de Copenhague", es decir, según la concepción de Louis Hjelmslev (las obras citadas son de los años 1928 a 1948) y Jens Holt (obras de 1943 y 1946), Alarcos define: "La significación del tiempo es una perspectiva, basada en el contraste remoto/irremoto y prospectivo/improspectivo" ${ }^{34}$. Son las mismas categorías que emplea Alarcos en $1949^{35}$, en el análisis que quizá convenga aludir aqui para ilustración de King en cuanto a los tiempos mencionados:

presente: no pasado, no futuro
imperfecto: pasado, no futuro
futuro: no pasado, futuro
condicional: pasado, futuro.

Los términos de Alarcos de "remotospectivo" o "pasado-temporal" aparecen resumidos aquí como "pasado"; y los términos de "prospectivo" o "futurotemporal" como "futuro".

En 1959, Alarcos observa que los tiempos presente y pasados "indican no la situación real del hecho en el decurso del tiempo, sino la perspectiva que el hablante adopta al considerarlo" ${ }^{36}$. Explica asi el presente histórico mediante la perspectiva de participación, frente al alejamiento del pasado, por ejemplo; el alejamiento también explica los usos corteses del imperfecto. Este rasgo semántico de participación existente en el presente según Alarcos corresponde al carácter fáctico que propone King, propiedad aparentemente pragmática que se desprende del rasgo semántico de perspectiva de presente. En otros términos, lo que es constante aparece como semántico en el análisis de Alarcos, mientras que en King tiene un carácter denominado pragmático. Resumamos como antes los tiempos mencionados, ahora según el citado enfoque de Alarcos:

Emilio Alarcos, Gramática estructural (1951), Madrid, Gredos, 1977, §s 58; véase también \& 105.

ss "Sobre la estructura del verbo en espafiol" (1949), en E. Alarcos, Estudios de gramática funcional del español, Madrid, Gredos, 1973; segunda edición, 1980, páginas $50-89$.

* "Cantaría: modo, tiempo y aspecto" (1959), en Estudios, págs. 95-108, pág. 106. 
presente: situación en el tiempo; perspectiva de participación imperfecto: situación en el tiempo; perspectiva de alejamiento futuro: posibilidad-posterioridad; perspectiva de participación condicional: posibilidad-posterioridad; perspectiva de alejamiento.

Como es sabido, Alarcos denomina "modalidad cero" a la propiedad del presente y del imperfecto de que "indican una situación en el tiempo", si bien es "el contexto o la situación lo que permite colocar la referencia de canto en la linea temporal de los acontecimientos" " ${ }^{37}$. Con el sistema de Alarcos los cuatro tiempos quedan caracterizados por dos rasgos, como también en el análisis de Waugh ${ }^{38}$ de un cuarto de siglo después que cita King $(1,5)$. El análisis de Waugh tiene la misma asignación de rasgos para el francés que el de Alarcos para el español, aunque definidos de manera diferente; el presente queda también descrito como tiempo no marcado para ambos rasgos.

El análisis del tiempo verbal realizado por King, en suma, está lleno de reminiscencias para los lectores de Hjelmslev y Alarcos, aunque haya que señalar que el texto no ofrezca pistas al respecto.

\section{El ASPECTO.}

\subsection{Aspecto en canté $y$ cantaba.}

El análisis del aspecto de King comienza con una definición provisional como "la noción semántica que permite al hablante describir una visión estructural de una situación" $(5.1,66)$, tras citar $(5.1,63)$ a Comrie ("diferentes maneras de ver la consistencia temporal interna de una situación" $\left.{ }^{39}\right)$. De nuevo, tras referirse a los análisis de Bull, Gili Gaya, y Stockwell, Bowen y Martin, King subraya el carácter subjetivo de la opción entre hablé y habla$b a$; de nuevo se aleja del cognocitivismo al proponer que los acontecimientos tienen una verdadera estructura real ("real world structure"), que no es el origen de la opción entre "pretérito" e "imperfecto" $(5.2,69)$. Los tipos de estativos que propone Guitart ${ }^{40}$ para explicar el aspecto le parecen demasiados; propone una distinción que considera "más básica" (5.2, nota 3): la posibilidad del hablante de "adoptar un punto de vista estructural externo acerca de una situación", de manera que el pretérito presenta la situación "en su

n "Cantaria", págs. 104 y 105, respectivamente.

Véase nota 16

* Bernard Comrie, Aspect, Cambridge, Cambridge University Press, pág. 3.

40 Jorge M. Guitart, "Aspects of Spanish aspects", en Margarita Suffer (ed.), Contemporary studies in Romance linguistics, Washington, Georgetown U. Press, 1978, páginas 132-168. 
totalidad", y el imperfecto más bien a la mitad; el pretérito es [+ perfectivo], y el imperfecto [- perfectivo] : hablaba presenta asi la situación como producida "a lo largo del tiempo", mientras que la concepción correspondiente a hablé no tiene en cuenta que la situación de hecho se produce a lo largo del tiempo $(5.2,73)$.

King considera que esta idea de perspectiva de la situación completa frente a exclusión de dicha perspectiva es característica de la teoría de prototipos, ya que la toma del análisis de Lunn ${ }^{41}$. Sin embargo, no son necesarios los prototipos: Isachenko " opone el aspecto imperfectivo, en que "nos encontramos en medio de un suceso, que no abarcamos con la mirada", al perfectivo, en que "abarcamos el acontecimiento como un todo", y los compara a ver un desfile como participante o desde la tribuna, respectivamente. King rechaza la utilidad de distinguir acontecimientos y estados $(5.1,63)$; sin embargo, parece probada (por ejemplo por Kuttert ${ }^{43}$ ) la utilidad de distinguir entre cuantificación sobre acontecimientos para el aspecto perfectivo frente a cuantificación sobre intervalos de tiempo para el imperfectivo ${ }^{4}$ : King está lejos de la idea de considerar las indicaciones flexivas del verbo como cuantificación, por ejemplo análoga a la de la determinación nominal ${ }^{45}$.

De nuevo, es pragmática para King la diferencia en Cervantes moría en 1616 y Cervantes murió en 1616, que toma del análisis de De Mello ${ }^{4}$ : con moría la visión [+ perfectiva] "está enmarcada pragmáticamente a lo largo de todo el año" $(5.2,77)$. Entre Conocía a su hermano y Conoci a su hermano, King acepta el análisis de Guitart "n de que conocí significa el comienzo del estado, mientras que conocía mantiene el significado léxico estativo: King lo califica como "excelente explicación de un uso pragmático concreto de la forma perfectiva" $(5.2,71)$, y más adelante $(5.3,93)$ afirma que "La forma imperfectiva puede potencialmente representar un estado (Lo conozco "I know him"), mientras que la forma perfectiva tiene un uso potencial noestativo u orientado hacia la actividad (Lo conoci, "I met him")". Además de la diferencia entre estado y acción que como hemos visto rechaza King, aparece la idea de lo pragmático como posible ("potencial").

4 Patricia Lunn, "The aspectual lens", Hispanic Linguistics, 2, 1985, págs. 49-61.

a Alexander V. Isac[h]enko, Die russische Sprache der Gegenwart, Teil 1, Formenlehre, Halle del Saale, 1962, pág. 350; citado por Werner Abraham, Diccionario de terminologia lingüistica actual (1974), Madrid, Gredos, 1981.

* Rainer Kuttert, Syntaktische und semantische Differenzierung der spanischen Tempusformen der Vergangenheit, Francfort del Meno, Lang, 1982.

* Véase J. Garrido, Lógica y lingüistica, Madrid, Síntesis, 1988, § 4.3.4.

4 Véase J. Garrido, "El lugar teórico del verbo en la descripción del español", en G. Wotjak y A. Veiga (ed.), La descripción del verbo español, Santiago de Compostela, Universidad, 1990, págs. 133-139; sobre la determinación, más abajo, \& 5.1 .

* George De Mello, "Some observations on Spanish aspect", Hispanic Linguistics, 3, 1989, págs. $123-129$.

17 Véase nota 40. 
Añadamos en cuanto al aspecto en cantó y cantaba que King no considera la opción de que el primero indique anterioridad y el segundo simultaneidad con el pasado, defendida por Bello y otros ${ }^{48}$.

\subsection{Aspecto de los tiempos compuestos: la orientación.}

En 1975, Alarcos modificaba su análisis de lo que consideraba "aspecto sintagmático" en 1949, pasanclo a caracterizar los tiempos compuestos mediante la "anterioridad" 49 . King, que no tiene en cuenta a Alarcos, sino a los ya citados Bull, Gili Gaya y Stockwell, Bowen y Martin $(4.2,45)$, cuyas explicaciones sobre la anterioridad de los tiempos compuestos enumera brevemente, denomina esta propiedad "orientación", y la define como la expresión de "una relación explícita de ordenación para la situación descrita", de manera que [+ anterior] corresponde a la ordenación de "la situación antes de otro tiempo o acontecimiento", que no se da en los tiempos simples, que tienen el rasgo de [- anterior] $(4.3,47)$. Asi, saliste no ordena la situación, mientras que has salido la ordena con respecto al "tiempo de la comunicación" $(4.4,52)$.

$\mathrm{La}$ indicación de que el hecho es reciente en ha muerto frente a murió "es una función del contexto"; "el contexto puede proporcionar numerosos matices de nociones pragmáticas" (4.4, 54 y 55$)$. Sin embargo, basta aplicar los rasgos semánticos que el propio King propone para considerar semántica la diferencia : presente con anterioridad en ha muerto frente a pasado en murió; el propio King lo hace $(4.6,59)$ para distinguir ¿Has fregado los platos? de ¿Fregaste los platos? A pesar de que repetidamente King insiste en que la cronología no determina el uso de los tiempos, considera que $\mathrm{He}$ estado en los Estados Unidos en 1993 es inadecuado porque he estado "tiene siempre [...] como interpretación pragmática potencial" "la medida de las veces que ocurre una acción hasta el presente (es decir, hasta antes del TDC [tiempo de comunicación])". De nuevo se considera pragmático algo que está "siempre”, si bien de una manera "potencial". Sin embargo, si concebimos el sig-

\footnotetext{
4. Bello, Gramática. \$ 624-633; E. Coseriu, Das romanische Verbalsystem, Tubinga. Narr, 1967, y “Aspect verbal ou aspects verbaux?", en J. David y R. Martin (ed.), La notion d'aspect, París, Klincksieck, 1980, págs. 13-25; Guillermo Rojo, "La temporalidad verbal en español", Verba, 1, 1974, págs. 68-149, y “Temporalidad y aspecto en espaffol, Lingüística Española Actual, 10, 1988, págs. 195-216; Alexandre Veiga, "Planteamientos básicos para un análisis funcional de las categorías verbales en espafiol", en G. Wotjak y A. Veiga (ed.), La descripción del verbo ex español, Santiago de Compostela, Universidad, 1990, págs. 237-257. \&4.

4 "Otra vez sobre el sistema verbal español" (1975), en Estudios, págs. 120-147,
} 
nificado de ha estado como anterioridad en el presente, no hay problema de ampliar el presente de la enunciación hasta 1933, como el propio King afirma repetidamente en su análisis del tiempo verbal presente.

\subsection{Aspecto perifrástico con estar y gerundio.}

Para describir la diferencia entre estoy cantando y canto King usa el término de descubierto (inglés overt. "público") que Hatcher ${ }^{50}$ emplea para el inglés. Se trata de la descripción de la acción en curso, desarrollándose, al descubierto $(5.3,87)$. Su análisis reproduce el trabajo anterior ${ }^{51}$; ya antes $(5.1$, n. 1,63$)$ remite a su otro artículo en colaboración con Suñer ${ }^{52}$ para rechazar el rasgo de estativo en este caso. Notemos que el término de descubierto recuerda a la distinción de Whorf ${ }^{53}$ entre categorias morfológicamente descubiertas (overt), con marcas que las explicitan, frente a categorias encubiertas (covert, "secretas"). Son términos en inglés relacionados etimológicamente con los participios del francés antiguo correspondientes a abierto y cubierto; sin embargo, King emplea como antónimo nonovert, "no descubierto". De nuevo surge el término de pragmática al tratar ejemplos como $\mathrm{Me}$ están escuchando y Me estaban escuchando, cuya "visión descubierta" según King "pragmáticamente representa [portrays] la situación como una actividad que se va desplegando en presencia del hablante" $(5.3,87)$.

Además de caracterizar las perífrasis de estar y gerundio, el rasgo de [+ descubierto] define semánticamente a estar frente a ser, [- descubierto] : "Pragmáticamente, [... Es abogado] es permanente"; Está de embajadora "podría implicar un cambio, un trabajo temporal, o cualquier otra implicación/inferencia pragmática que sea compatible con la interpretación de duración explícita proveniente de la caracterización en curso y en desarrollo de la situación" $(5.4,107)$. También aquí considera King pragmáticos los valores que según él provienen de la relación entre el dato morfológico y cualquier otra indicación, léxica o de otro tipo, como el propio King expresa en su conclusión: "la interacción entre significado sistemático y significado léxico $[\ldots]$ permite explicar cierta variación en el uso pragmático" $(5.5,129)$. Como veremos (7.1), más que de interacción se trata de que la información morfológica forma parte de la estructura sintáctica.

\footnotetext{
* Anna Granville Hatcher, "The use of the progressive form in English", Language, 27, 1951, pags. 254-280.

s1 "The meaning of the progressive in Spanish and Portuguese"; véase nota 3.

sa "On the notion of stativity in Spanish and Portuguese"; véase nota 3.

ss Benjamin Lee Whorf, "Categorías gramaticales" (1937), en B. L. Whorf, Lenguaje, pensamiento y realidad (1956), Barcelona, Barral, 1971, págs. 105-120.
} 


\section{EL MODO.}

\subsection{Modo y relevancia.}

En el análisis del modo aparece la mención a la teoria de la relevancia de Sperber y Wilson tal como la aplica Lunn ${ }^{54}$; para King es dudoso que el uso de votara se deba al deseo del hablante de asignar menor relevancia a la información :

Se trata de un nuevo desafio al poder central $[\ldots]$ que se consideraba inevitable después de que, el 31 de marzo, más del $90 \%$ del censo votara a favor de la secesión [...]. (Supresiones de King.)

La declaración es consecuencia del plebiscito del 31 de marzo, en el que $90 \%$ del censo votó por establecer la independencia.

Según King es el presente es el que requiere indicativo, frente al pasado consideraba, con el cual "se usa el pasado de subjuntivo como forma alternativa de expresión de anterioridad” $(6.1,134)$.

$\mathrm{El}$ modo "es la noción semántica que permite al hablante representar una situación como parte objetiva del mundo real", con el rasgo de [+ objetivo], frente al rasgo de [ - objetivo], que "significa que el hablante hace otra cosa que describirla como hecho objetivo" $(6.1,141)$. King $(6.1,137)$ se apoya en el análisis de Bergen ${ }^{55}$, según el cual el hablante considera la proposición como "hecho objetivo" con el indicativo, mientras que el subjuntivo "expresa una reserva subjetiva por parte del hablante (o el actor) acerca de la realidad de dicha proposición". Como observa el propio King $(6.4,141)$, sus términos no son nada nuevo en el análisis del modo.

\subsection{La suposición contextual como alternativa en el análivis del modo.}

Basándose en que "creer está orientado hacia el creer y dudar está orientado hacia el dudar" $(6.2,153)$, King explica No dudo que sea más listo por la capacidad de [- objetivo] de arrojar duda, de manera que "es lógico" que el hablante exprese tal reserva cuando usa dudar. Afiade además (6.2, 154) que los únicos hispanohablantes que ha encontrado que defiendan que

A Dan Sperber y Deirdre Wilson, Relevance. Communication and cognition, Oxford, Blackwell, 1986; Patricia Lunn, "Spanish mood and the prototype of assertability", Lingemistics, 27, 1989, págs. 687-702.

ss John J. Bergen, "One rule for the Spanish subjunctive”, Hispania, 61, 1978, páginas 218-234, pág. 221. 
se dice No dudo que es más listo son profesores de español que llevan años metiéndoles esa idea a los estudiantes ("drilling this notion into the head of their students". con una agradable anfibologia de drill entre el ejercicio repetition y el perforarles las cabezas).

Se puede echar falta aqui la distinción entre negación lingüistica y metalingüistica, entre negación de un hecho y negación de una formulación lingüistica de un hecho ${ }^{56}$. En otros términos, con el subjuntivo se considera el dato como idea presente en calidad de información contextual, es decir, como suposición contextual ${ }^{5}$. La existencia de esta suposición es constante; lo que depende de las circunstancias es el origen que se le da. Por ejemplo, se puede atribuir al interlocutor. Con el indicativo, se trata el dato como propuesto, es decir, como proposición objeto de aserción. King se acerca a este enfoque cuando observa que en casos como No cree que participó "la visión [+ objetiva] del indicativo describe la opinión contraria [counteropinion] del hablante". mientras que en No cree que participara "la visión [- objetiva] lel subjuntivo $\lceil\ldots\rceil$ repite la aserción negativa del sujeto" $(6.2 .151)$. Sin embargo, no queda claro cómo da lugar el modo a esta diferencia.

Otra alusión a consideraciones normativas es la observación sobre $l Q u e ́$ bueno que has llegado tan puntualmente!: "Prescriptivamente se deberia usar el subjuntivo ya que qué bueno implica un comentario por parte del hablante", aunque de hecho se usa con frecuencia el indicativo $(6.1,140)$. Lo interesante es considerar prescriptiva tal relación, aunque a continuación se rechace.

En Aunque sea mi hija, la encuentro muy guapa, King atribuye al rasgo [- objetivo] la menor relevancia, siguiendo a Lunn. o carácter marginal, de acuerdo con Lavandera $\$$, que el hablante asigna al dato de ser su hija mediante el subjuntivo $(6.3,161)$. Me gustaría destacar que la novedad del análisis de Sperber y Wilson basado en el de Grice consiste precisamente en explicar cómo se llega a asignar menor relevancia al dato ${ }^{50}$; no parece, pues, suficiente limitarse a considerar que el dato es una consecuencia del rasgo [ - objetivo]. En cualquier caso, se podría aplicar al subjuntivo el concepto de supo-

6 Oswald Ducrot, Decir y no decir (1972), Barcelona, Anagrama, 1986, cap. 2.

s7 Véase J. Garrido. "Operadores epistémicos y conectores contextuales". Diálogos Hispánicos de Amsterdam, 12, 1993, págs. 5-50, \& 3.5 .

* Beatriz R. Lavandera, "Shifting moods in Spanish discourse", en Flora Klein [-Andreu] (ed.), Discourse perspectives on syntax, Nueva York, Academic Press, 1983, págs. 209-236.

* Deirdre Wilson y Dan Sperber, "Sobre la definición de la relevancia" (1986), y Paul H. Grice, "Lógica y conversación" (1975), ambos en Luis M. Valdés (ed.), La brisqueda del significado, Madrid y Murcia, 1991, en Tecnos y Universidad de Murcia, páginas 583-598 y 511-530, respectivamente; véase también el libro de Sperber y Wilson citado en la nota 54 . 
sición contextual que he mencionado en el penúltimo párrafo ${ }^{\infty}$ : con sea se hace frente a una posible observación del interlocutor, mientras que con es se expresa un dato. En un caso se trata de una suposición contextual (una idea presente o introducida en el contexto, atribuida al interlocutor, al propio hablante, o a conocimientos generales o particulares de ambos), y en el otro es una proposición afirmada explícitamente. Tanto el carácter de suposición atribuida como el de proposición afirmada son propiedades semánticas.

King admite que la especificidad en las construcciones de relativo sea gradual, como propone Morgan ${ }^{61}$, pero la circunscribe a su concepto de pragmática $(6.4,165)$ y la define como "existencia del antecedente del nombre como hecho objetivo" $(6.6,175)$. De todos modos, considera que en Son los sueños más maravillosos que en ni vida haya podido tener "Los sueños son especificos" $(6.4,167)$, cuando en realidad son específicos los sueños que de hecho ha tenido (descritos como sujeto tácito de son), y son los más maravillosos de un conjunto que no es específico. Efectivamente, la construcción comparativa desdobla la cuantificación de modo que con el artículo (los sueños) se cuantifica sobre un conjunto contextual relacionado con el mundo cognoscitivo del hablante (carácter específico) mientras que con el comparativo (los más maravillosos que haya podido tener) se cuantifica sobre un conjunto de base sin tal relación ${ }^{62}$ : es algo semejante al efectivo desdoblamiento en Los sukños los más maravillosos que en mi vida haya podido tener. El modo subjuntivo da al conjunto sobre el cual se cuantifica (el de los más maravillosos) el carácter cle suposición contextual, de modo que no esté relacionado con el mundo cognoscitivo del hablante, sino con lo que pudria pensar el hablante o mejor el oyente acerca de sueños maravillosos del hablante.

En cuanto al imperativo, el hecho de que una oración declarativa en indicativo sirva para comunicar indirectamente una orden o sugerencia lleva a King a afirmar que no es necesaria "una noción semántica propia para el imperativo" $(6.5,170)$. Notemos de paso que King cita como ejemplo Deseara hablar con usted (en 3.5, 31), sin reparar en la observación de Gili Gaya ${ }^{63}$ de que este uso es posible con los verbos querer, deber y poder. (Esto recuerda al modo en que King incorpora sin comprobarlo el ejemplo $\mathrm{Me}$ fui que Gili Gaya ${ }^{\star 4}$ da como expresión chilena "pronunciada antes de irse para de-

wo Con ello se aborda lo que para otros, por ejemplo A. Alonso-Cortís según observación hecha personalmente, es un fenómeno de discurso; véase también Ångel Manteca Alonso-Cortés, Gramática del subjuntivo, Madrid, Cátedra, 1981, § 2.6.

61 Terrell Morgan, Spanish mood and the pragmatics of adjective clauses, University of North Carolina at Chapell Hill, 1983, tesis de máster.

62 Véase J. Garrido, Aspectos semínticos y sintácticos del artículo en español (1984), Madrid, Universidad Complutense, 1987, págs. 761-791, y Elementos de análisis lingïistico, Madrid, Fundamentos, 1991, págs. 121-137; véase también más abajo, \$ 5.1 .

a Curso, \& 129.

a Curso, \& 122. 
notar la inninencia de la acción”.) Precedido de quizás, el ejemplo de King $(6.5,171)$ „Pudiera servirme un poco más de arroz? sí podria emplearse como petición cortés. En nota $(6.5$, n. 8,170$)$, King pone en duda la necesidad de considerar el imperativo como modo, ya que "el imperativo es una noción pragmática compartida por el subjuntivo y el indicativo".

\section{El rasgo DE PARTITIVIDAD.}

\subsection{El determinante: partitizidad frente a cuantificación contextual.}

En cuanto al artículo, King $(7.2 .2,181)$ parte de la diferencia de Bull os entre la indicación de "totalidad (uno único)" en el y la de "partitividad (uno de muchos)" en un ${ }^{66}$. Toma de Suñer ${ }^{67}$ el rasgo de [ - individualizado] para los sustantivos sin artículo frente al de [ + inclividualizado] para los sustantivos con determinante. Según King $(7.2 .2,185)$, el análisis es compatible con el de Alonso \&s si bien éste "trata de caracterizar la diferencia en términos de la realidad" (cabe preguntarse si "las valoraciones subjetivas y categoriales de las cosas" que propone Alonso ${ }^{\circ}$ para el nombre sin artículo encajan en esta valoración de King). Son pragmáticos para King los dos fenómenos que señala Bull ${ }^{\text {ro }}$ : el primero es el establecimiento de foco mediante un y su mantenimiento mediante $e l$ (en un análisis y con un ejemplo semejantes al de Alonso " de que un sirve para "introducir" un objeto mientras que con el "se le sigue nombranlo"; King no alude a este análisis de Alonso). El segundo fenómeno pragmático es la diferencia en $e$ el entre la to-

os William E. Bull, Spanish for teachers: applied lingwistics, Berkeley y Los Angeles, University of California Press, 1965, pág. 215.

* Se trata de la diferencia propuesta para el francés por Guillaume en 1919 y aplicada a varias lenguas posteriormente, entre ellas el inglés; véase Gustave Guillaume, Le probleme de larticle et sa solution dans la langue française (1919), Nizet y P. U. Laval, París y Quebec, 1975; J. Garrido, “Actualización, sustantivación, cuantificación y contexto en el artículo español en la teoría lingüística del siglo $x^{\prime}$ ", en prensa en las Actas do XIX Congreso Internacional de Lingüistica e Filoloxía Románicas (Santiago de Compostela, 1989), La Corufia, Fundación P. Barrié de la Maza. Véase en la nota 78 la referencia a la obra de Wilmet, que parte de esta idea de Guillaume en su análisis de la determinación.

o7 Margarita Suñer, On presentationals: Syntax and semantics of a Spanish sentence type, Washington, Georgetown UP, 1982.

* Amado Alonso, "Estilistica y gramática del artículo en espafiol" y "Adición en 1951", en A. Alonso, Estudios lingüisticos. Temas españoles, Madrid, Gredos, 1951, págs. 125-149 y 149-160.

* "Estilistica", pág. 134.

* Spanish for teachers, págs. 220 y 215.

$n$ “Adición", pág. 160. 
talidad genérica (observada por Bello ${ }^{12}$ ) y la totalidad de uno único; King, como antes Alonso (pero de nuevo sin citarle), atribuye la diferencia al contexto $(7.2 .2,188)$.

El problema sigue siendo cómo tienen lugar estas diferencias, que para King simplemente se deducen "pragmáticamente" de los rasgos [+ partitivo] y [ - partitivo]. Como alternativa a esta concepción de rasgos, se puede entender la determinación nominal como cuantificación del conjunto descrito por el resto del sintagma (perros verdes) con respecto a un conjunto contextual que se reconstruye o construye en el modelo cognoscitivo del mundo que tiene el hablante, de modo que $e l$ indique la coincidencia total (los perros verdes) y un parcial (unos perros verdes) entre ambos conjuntos, mientras que la ausencia de artículo supone la falta de esta relación cuantificadora con el conjunto contextual; el neutro es también cuantificación totalizadora (lo verde), y se opone al carácter humano o animado (los verdes, "los ecologistas") o a la anáfora concordante con un sustantivo de los otros pronombres personales (Voy a llevar corbata, la verde, por ejemplo) ${ }^{73}$.

Para los demostrativos y posesivos King adopta los rasgos de próximo y remoto de Lyons ${ }^{74}$, añadiendo los de pronombre, demostrativo y posesivo. Como es tradicional, por ejemplo en Bello y Alarcos (aunque King se lo atribuye a Bull y a Suñer), los demostrativos y posesivos tienen además el mismo rasgo semántico que el artículo definido, [- partitivo] en los términos de King $(7.2 .3,194)$.

\subsection{El número nominal.}

En el análisis del número nominal King aplica la diferencia propuesta por Langacker ${ }^{75}$ entre sustantivos discontinuos o contables, que designan regiones concebidas como acotadas; sustantivos continuos o de materia, que designan regiones no concebidas asi; y sustantivos abstractos, cuyas regiones designadas pueden estar acotadas o sin acotar. Para King, el plural cambia el rasgo de [ - partitivo] en coche, y de [+ partitivo] en vino y honor, a [+ partitivo] (coches) y a [- partitivo] (vinos, honores), respectivamente $(7.3,200)$. Se pasa de totalidad a partes iguales, o se crean totalidades, respectivamente. Pragmáticamente, "el rasgo [ \pm partitivo] se transforma en el singular/plural del mundo real"; "el contexto debe determinar el referente pragmático concreto de los valores de $[ \pm$ partitivo]" $(7.3,201)$.

72 Gramática, \& 270.

73 Sobre estas ideas véase J. Garrido, Elementos, págs. 89-190.

4 John Lyons, Introducción en la lingüistica teórica (1968), Barcelona, Teide, 1975.

70 Langacker, Foundations, págs. 203-208 (véase nota 19). 
Aqui resulta inevitable recordar (a diferencia de King) el análisis de Fernández Ramirez ${ }^{76}$ de los nombres de cosa (categoria primera) y los nombres de sustancia y abstractos (categoría segunda), según el cual los plurales de la categoria primera pueden también designar las clases (Hay clásicos y clási$\cos )$, mientras que la categoría segunda puede usarse también en plural para los tipos o clases (cafés, tés, chocolates, rótulo de almacén de ultramarinos; ungustias $y$ alegrias).

\subsection{La posición del adjetivo.}

El rasgo de partitivo se aplica a la posición del adjetivo, además de al determinante $\mathrm{y}$ al número nominal. Bull ${ }^{n}$ propone que la adición del adjetivo al sustantivo se interpreta como totalidad o como partitividad; para King $(7.4,207)$, en estupenda película " $\mathrm{La}$ anteposición concibe el referente $[\ldots]$ como una totalidad": mientras que la posposición en película estupenda lo concibe "como una parte de una totalidad mayor [+ partitivo], admitiendo la relevancia de otros". Se trata, claro está, de la tradicional diferencia entre modificación explicativa y especificativa (o restrictiva).

Según King, esta diferencia concebida como rasgo semántico pragmáticamente da lugar al contraste: la encantadora Italia, [- partitivo], es "pragmáticamente no contrastiva", mientras que la Italia encantadora, [+ partitivo], es "pragmáticamente contrastiva por posición". King llega al extremo de considerar la entonación contrastiva como "pragmáticamente contrastiva" $(7.4,210)$. Detrás de ello se intuye el efecto de la modificación especificativa del adjetivo pospuesto, que restrinje y por tanto permite el contraste (la Italia encantadora frente a la Italia no tan encantadora). Sin embargo, es posible limitar la explicación en la línea de Bull al terreno de la semántica, aplicando por ejemplo al español el análisis del francés que hace Marc Wilmet: el orden la Italia encantadora es el no marcado, que linita la alusión a aquella parte de Italia que es encantadora, mientras que la encantadora Italia es el orden marcado, en que la propiedad de ser encantadora es una de las propiedades incluidas en Italia ${ }^{78}$.

La posición del sujeto con respecto al verbo es también explicable para

76 Salvador Fernández Ramírez, Gramática española (1951), Madrid, Arco, 1986, tomo $2.1, \& 94$.

77 Sparish, págs. 226-227.

78 Marc Wilmet, La détermination nominale. Quantification et charactérisation, $\mathrm{Pa}$ rís, Presses Universitaires de France, 1986. En el capítulo quinto, dedicado a la posición del adjetivo calificativo en francés, analiza un conjunto complejo de factores de toda índole que aclaran los diferentes tipos de orden marcado, aplicables al ejemplo la encantadora Italia. 
King $(7.4,217)$ como [+ partitivo] en la posposición del sujeto, en que "el sujeto es visto como parte de un conjunto más amplio"), y como [- partitivo] en la anteposición ("el sujeto es visto como una totalidad"). Naturalmente, son posibles (y más plausibles) otras explicaciones, por ejemplo las de Torrego, Fernández Soriano o Mallén ${ }^{79}$, a partir de la idea de que la inversión y en general el carácter de tema, frente al de rema, como exponen Hernanz y Brucart ${ }^{\infty}$, son propiedades sintácticas (que, por serlo, tendrán su consiguiente efecto en la estructura semántica oracional y, como veremos brevemente, en la estructura textual).

\section{Preposición $a$ y complemento diRecto: RASGo FRente a marca.}

Un ejemplo tomado de Bello (Hemos de matar en los gigantes a la soberbia $[\ldots])$ le sirve a King para rechazar que la "forma morfológica libre" a confiera el estatuto de humano. De Fish ${ }^{81}$ acepta la idea de que esta preposición puede ser un "símbolo de estatus", de igualdad o superioridad frente al sujeto. Con la preposición el hablante asigna igual estatus "al objeto directo", sin ella le niega "igual rango" $(8.2,229)$. "Pragmáticamente", de nuevo, el igual rango "se interpreta como ser humano concreto en el mundo, $\tan$ humano e individualizado como el hablante", en el clásico Busco a un médico, frente al "no individualizado y por tanto no concreto [specific]" de Busco un médico $(8.2,231)$. Así se explica la "despersonificación pragmática" o cosificación de Llevaron los presos al cuartel ${ }^{82}$ y, siguiendo a Suñer ${ }^{83}$, la ausencia de $a$ en Hay hombres que para nada sirven, ya que el complemento directo sintáctico "no puede ser semánticamente objeto directo" por no existir agente ni paciente $(8.2,236)$.

Aquí se puede echar en falta un tratamiento de sintaxis de prototipos, en la línea de Hopper y Thompson y de Lazard, y, para el español, Roegiest ${ }^{84}$ :

79 Esther Torrego, "On inversion in Spanish and some of its effects", Linguistic Inquiry, 15, 1984, págs. 103-129; Olga Fernández Soriano, "Tematización, dislocación y focalización en castellano", en C. Martín Vide (ed.), Actas del IV Congreso de Lenguajes Naturales y Lenguajes Formales, Barcelona, PPU, 1989, págs. 595-607; Enrique Mallén, "Subject topicalization and inflection in Spanish", Theoretical Linguistics, 19, 1992, págs. 179-208.

* María Luisa Hernanz y José María Brucart, La sintaxis, 1, Barcelona, Crítica, 1987, capítulo 3.

81 Gordon T. Fish, " $A$ with Spanish direct objects", Hispania, 50, 1967, págs. 80-85, pág. 80 .

82 King traduce cuartel por jail, es decir, cárcel.

os On presentationals; citado en la nota 67.

a P. J. Hopper y Sandra A. Thompson, "Transitivity in grammar and discourse", Language, 56, 1980, págs. 251-299; G. Lazard, "Actance variations and categories of the object", en F. Plank (ed.), Objects: tonvards a theory of grammatical relations, Nueva 
un objeto prototípico, el paciente por excelencia, inanimado e indefiniđo, no requiere marca, mientras que un objeto que se sale del prototipo por sus rasgos de humano y definido, individualizado y autónomo, al ser susceptible de ser él el agente, requiere la marca $a$ para ser distinguido del sujeto: se marca como afectado lo que de otro modo podria ser tomado por agente (Maria saluda a Pedro). También cuando hay dos sintagmas de rasgo inanimado los dos pueden ser agente o paciente (La preposición caracteriza al objeto); por eso se marca uno como afectado en lugar de como paciente, mediante la preposición ${ }^{85}$.

King $(8.2,239)$, con ejemplos de Weissenrieder ${ }^{86}$, rechaza que en casos con dos partilipantes inanimados (El carro negro alcanzó al azul) la preposición sirva para desambiguar. King cita la explicación de Weissenrieder, cercana a la del objeto prototípico mencionada, de que la preposición "puede aparecer más predeciblemente cuando hay igualdad semántica relativa (entre sujeto y objeto) que como marca de ambigüedad semántica potencial" $"$. Para encajarla en su hipótesis, King afirma que el rasgo de igualdad de rango puede desdoblarse en "perspectiva del hablante y perspectiva del sujeto", como en el modo, de manera que indique [ + igual rango] en Tiene a su hijo en sus brazos frente a [- igual rango] en Tiene un hijo. Sin embargo, los mencionados factores de naturaleza prototípica parecen explicar mejor el uso de la preposición que este desdoblamiento del rasgo semántico. King termina añadiendo en nota $(8.3$, n. 5,243$)$ que el artículo de Weissenrieder apareció después del suyo de 1984, y que cree que el análisis de Weissenrieder, continuado en 1990 y 1991 , "apoya y clarifica" el suyo.

\section{Conclusión: pragmática fuera o dentro de la gramática.}

\subsection{El lugar del significado morfológico en la gramática.}

King añade un capítulo $(9,245-275)$ de comparación con el inglés. Conviene destacar la inexistencia según King de modo en inglés, así como, natu-

York, 1984, Academic Press, págs. 269-292; Eugeen Roegiest, "La tipología sintáctica del objeto transitivo en español", Verba, 17, 1990, págs. 239-248.

83 Véase en J. Garrido, Idioma é información, Madrid, Sintesis, 1994, § 9.4.4, un análisis de este tipo de las marcas de complemento directo e indirecto y de su relación con el leísmo.

86 Maureen Weissenrieder, "Exceptional uses of the accusative a", Hisparia, 68, 1985, págs. 393-398.

87 Weissenrieder, "Exceptional uses”, pág. 396.

88 King se refiere a su artículo "The semantics of direct object $a$ in Spanish" (véase la nota 2); los de Maureen Weissenrieder son "Variable uses of the d[irect] o[bject] marker $a$ ", Hispania, 73, 1990, págs. 223-231, y "A functional approach to the accusative $a "$, Hispania, 74, 1991, págs. 146-155. 
ralmente, la de preposición $a$ (figura como tal ausencia de $a$, "direct object $a$ : no", en la tabla de $9.2,246$ ), además de las conocidas diferencias en la flexión verbal y en los determinantes, que King analiza según los conceptos anteriormente tratados, y en el caso del verbo inglés, según su artículo de $1983^{89}$. A continuación figura el capitulo de conclusión (10, 277-283).

$\mathrm{El}$ argumento de que el significado representado morfológicamente es diferente del significado léxico se vuelve contra King por dos razones. En primer lugar, en lugar de simplemente reflejar la realidad ("La semántica léxica representa [depicts] los fenómenos del mundo real", escribe King; 10, 280), el significado léxico organiza cognoscitivamente los datos disponibles. En un enfoque realista, la realidad aparece reproducida sin más en el léxico; en un enfoque cognoscitivista es el conocimiento de la realidad lo que aparece reproducido en el léxico. De este modo, desaparece la tajante diferencia de naturaleza entre significado representado léxicamente frente a significado representado morfológicamente.

En segundo lugar, la información representada morfológicamente, como cualquier información, se combina con el resto de la información de la expresión lingüística. El tiempo y el aspecto, por ejemplo, no son explicables como unidades indivisibles, como rasgos simples; más bien se trata de unidades construidas a partir de unidades inferiores, y entre estas últimas figuran las que corresponden a la flexión verbal. $\mathrm{Si}$ se concibe la estructura sintáctica como una red jerárquica de nudos de sintagmas que a su vez constituyen sintagmas, el tiempo es un nudo de esa red, y el aspecto probablemente otro, a su vez componentes de la categoría de la flexión verbal ${ }^{90}$. Es necesario tener en cuenta este carácter de componente de la estructura sintáctica que tiene la información morfológica.

Veamos un ejemplo. Según King $(3.7,40)$, si se enuncia Pilar me dice que $v a$ al cine anoche el enunciado parece inadecuado; pero "Se puede uno fácilmente imaginar una situación en que la hablante llama muy nerviosa a alguien: Me he enterado de que anoche Pilar va al cine $y$ antes de entrar encuentra $a[\ldots]$ ". Notemos que King define su concepto de situación como "cualquier situación del mundo real descrita por el verbo", incluyendo "intenciones así como acontecimientos, estados y procesos" (5.2, n. 2, 68). La conclusión del ejemplo para King $(3.7,40)$ es que "no siempre se puede hacer juicios claros acerca de cuándo se usará una forma en lugar de otra". Sin embargo, en el ejemplo, la flexión verbal de he enterado explica el uso de va y encuentra construido con anoche. No se puede explicar como "uso pragmático" esta construcción, basándose en que "pragmáticamente la perspectiva

29 Véase la nota 2.

90 Como muestra, véase E. de Miguel, El aspecto, $\$ 1.5 .2$ (citado en la nota 12). 
temporal representa los acontecimientos como si estuvieran ocurriendo en el momento de hablar" $(3.7,40)$.

\subsection{Pragmática frente a gestión semántica de la información.}

Por último, King cita el clásico ejemplo de No tengo dinero como negativa a ${ }_{6}$ Vas al cine con nosotros? para argumentar que "el papel de la pragmática más allá de la forma lingüística no puede pasarse por alto" $(10,282)$. Como se ha ido viendo anteriormente, no basta con declaraciones de interés por la pragmática ni con afirmaciones de que los rasgos semánticos tienen "potencial pragmático" $(10,282)$. Es esta una semántica procrusteana (que expulsa de la estructura semántica todo lo que le cabe en ella). La pragmática queda fuera, caracterizada además por la imposibilidad de predecir el contexto y las interpretaciones ("la impredecibilidad de forma y contexto", 3.7, 40 ; "Cada interpretación pragmática es variable y no predecible", 7.4, 209).

El propio King califica la relación entre semántica y pragmática como de complementaria, puesto que "en cuanto a la forma morfológica, hay diferencia entre contenido semántico y potencial pragmático" (10,282). La relación de complementariedad supone, sin embargo, que los fenómenos considerados pragmáticos sigan quedando fuera del significado en cuanto información representada de manera constante por las expresiones o sus componentes. En términos de Escandell ${ }^{91}$, la pragmática, como la gramática, "se ocupa de datos lingüísticos $[\ldots]$; pero recordemos que siempre hace referencia a los elementos extralingüisticos que configuran la situación de emisión, así que, en este sentido, se diferencia de ella".

Frente a estas concepciones de complementariedad, hay posibilidades de una semántica flexible ${ }^{92}$ en que el acceso al contexto está determinado lingüisticamente, $y$ en que los aspectos de otro modo considerados pragmáticos se integran en la información asociada de manera constante a las expresiones, es decir, en el significado y, por tanto, en la gramática ${ }^{93}$. Para ello es necesario, en primer lugar, considerar como contexto exclusivamente la información utilizada lingüísticamente; en segundo lugar, hay que tener en cuenta que hablante y oyente emplean un modelo mental de su interlocutor, es decir, una idea de qué información tiene y puede emplear. Además de tratar infor-

01 María Victoria Escandell Vidal, Introducción a la pragmática, Barcelona y Madrid, Anthropos y Universidad Nacional de Educación a Distancia, 1993, § 14.4, pág. 270.

* Véase J. Garrido, "Semántica flexible", en Actas del Congreso de la Sociedad Española de Lingüistica, XX Aniversario, Madrid, Gredos, 1990, págs. 497-505.

os Véase J. Garrido, Idioma e información, Madrid, Síntesis, 1994, § 7.4.4. 
mación acerca de la realidad, hablante y oyentes gestionan esa información, es decir, dan y usan orientaciones acerca de cómo debe ser procesada. Esta información sobre la gestión de la información no es algo extralingüístico. $\mathrm{Si}$ se considera una intención el describir un hecho de referencia pasada mediante el tiempo verbal presente (Me he enterado de que anoche va al cine Pilar y $[\ldots])$, o el atribuir una información al interlocutor, o a sí mismo, de manera que a continuación se rechace (aunque sea mi hija), estos datos llamados intenciones no se pueden considerar extralingüísticos ni extragramaticales.

Con respecto al presente histórico, el propio King $(3.3$, n. 2, 23) cita el análisis de Silva-Corvalán ${ }^{\text {r }}$ según el cual en el uso del presente con referencia pasada hay un mecanismo de evaluación interno del hablante. Recordemos la opción de la gestión de información: los datos representados lingüisticamente tienen que ver con hechos o situaciones de nuestro conocimiento de la realidad (información veritativa, susceptible de ser verdadera o falsa). Los datos lingüísticos también tienen que ver con el modo en que el interlocutor debe encajarlos en su modo cognoscitivo de la realidad, con el modo en que debe valorarlos. Cuando hay datos expresados lingüísticamente que permiten entender la referencia temporal como pasado, el presente añade la evaluación (por ejemplo, que el acontecimiento no se debe considerar como lejano en el tiempo).

En otros términos, el presente (con respecto al pasado) añade una información que es tan constante como la de la referencia temporal. Además, para que se pueda entender sin error como referido a un hecho del pasado, el presente requiere que haya una información adicional que asegure la referencia temporal (en el ejemplo, el anoche y la flexión verbal de he enterado, relacionados entre sí según una cierta estructura sintáctica). A la inversa, si no hay esta información adicional (por ejemplo anoche), la evaluación (de ser pasado pero tomarse como muy próximo) aparece para que la referencia temporal de la expresión no sea contradictoria con el resto. Se entiende el tiempo verbal de presente como referido a un hecho del pasado muy reciente cuando no hay más remedio, cuando solo con esta información de evaluación es coherente la expresión con el resto de la oración o del texto. Por consiguiente, este dato acerca de la gestión de la información es también parte de lo que entendemos por lengua, y por tanto su descripción forma parte de la gramática.

King concluye esperando haber mostrado que "los significados de las formas morfológicas suponen algo más que la expresión del uso de una determinada forma en un cierto contexto, y que entre la aparente confusión sí que

\footnotetext{
a Carmen Silva-Corvalán, "Tense and aspect in oral Spanish narrative: context and meaning", Language, 59, 1983, págs. 760-779.
} 
hay un principio organizador" $(10,283)$. En su minucioso y cuidado estudio, Larry King muestra interés y entusiasmo; al lector que lo haya seguido con la misma actitud, le puede sin embargo rondar la impresión de que efectivamente hay en estos fenómenos diversos un principio organizador, pero quizás no sea este de King, o por lo menos no exactamente en sus términos, sino tal vez en otros semejantes a los propuestos aqui como alternativa. 